\title{
$T_{1 p}$ Mapping of Pediatric Epiphyseal and Articular Cartilage in the Knee
}

\author{
Jared Guthrie Cobb, MS ${ }^{1,2,{ }^{*},}$ J. Herman Kan, MD $^{3}$, and John C. Gore, PhD ${ }^{1,2,4}$ \\ ${ }^{1}$ Vanderbilt University Department of Biomedical Engineering, Nashville, Tennessee, USA \\ ${ }^{2}$ Vanderbilt University Institute of Imaging Science, Nashville, Tennessee, USA \\ ${ }^{3}$ Texas Children's Hospital Department of Radiology, Houston, Texas, USA \\ ${ }^{4}$ Vanderbilt University Department of Radiology and Radiological Sciences, Nashville, \\ Tennessee, USA
}

\begin{abstract}
Purpose-To evaluate the feasibility of measuring $\mathrm{T}_{1 \rho}$ values in epiphyseal cartilage in children, we have conducted a novel study of spin locking techniques. Adult articular cartilage has been widely studied with spin locking techniques by magnetic resonance imaging. However, no results are available for in vivo $T_{1 \rho}$ imaging of developing cartilage.
\end{abstract}

Materials and Methods-Ten volunteers of age $6 \pm 3$ years were recruited to have $T_{1 \rho}$ mapping performed on the knee at the conclusion of their clinical study. $\mathrm{T}_{1 \rho}$ maps were generated using a spin-lock cluster followed by a fast spin-echo imaging sequence. Regions of interest (ROIs) were placed in non-load-bearing (NLB), load-bearing (LB), and articular cartilage.

Results-Student's t-tests were performed to compare means among the ROIs. Mean $\mathrm{T}_{1 \rho}$ for epiphyseal and articular cartilage was $49.8 \pm 9$ and $76.6 \pm 7 \mathrm{~ms}$, respectively. $\mathrm{LB}$ and NLB $\mathrm{T}_{1 \rho}$ vales were $47.1 \pm 9.5$ and $52.5 \pm 9 \mathrm{~ms}$, respectively. Significant differences were found in $T_{1 \rho}$ values between epiphyseal and articular cartilage layers $(P=0.0001)$. No difference in $\mathrm{T}_{1 \rho}$ was observed between NLB and LB layers. A modest trend was also noted for epiphyseal and articular cartilage regions with age.

Conclusion-It is feasible to quantify differences in epiphyseal and articular cartilage layers with SL techniques. $\mathrm{T}_{1 \rho}$ holds promise as a noninvasive method of studying normal and abnormal developmental states of cartilage in children.

\section{Keywords}

cartilage; $\mathrm{T}_{1 \rho} ;$ spin lock; $3 \mathrm{~T}$

\begin{abstract}
MR imaging is a uniquely powerful tool for investigating the structure and composition of the growing skeleton. MR imaging does not use ionizing radiation so it can safely be used to study the growth and development of structures such as epiphyseal and articular cartilage in children. Such studies may provide a greater understanding and early detection of perturbations in normal cartilaginous development that may leave permanent sequelae in the skeletally mature patient.
\end{abstract}

(C) 2013 Wiley Periodicals, Inc.

*Address reprint requests to: J.G.C., VUIIS, AA 3112 MCN, 1161 21st Avenue South, Nashville, TN 37232-2310. jared.g.cobb@vanderbilt.edu. 
Cartilage undergoes dramatic changes during the first 10 years of life but is more difficult to image than other tissues because it is present only in relatively thin layers, has relatively high density, and features a varied composition. Articular cartilage is composed of a semisolid matrix of water $(65-80 \%)$, collagen $(15-20 \%)$, proteoglycan $(\sim 5 \%)$, and other proteins $(\sim 2 \%)$ (1). Proteoglycan (PG) content is of particular interest because of its role in epiphyseal and articular cartilage structure and development. A subset of PGs, glycosaminoglycans (GAGs), are highly negatively charged macromolecules that make up a large percentage of cartilage PG content and contain a high concentration of sulfate groups that strongly bind to water molecules (2). This binding affinity helps to support collagen's triple-helical fiber structure which serves a variety of biomechanical functions in vivo, including shock absorption, flexibility, and smoothing of joint motions (3).

Epiphyseal cartilage is found at the ends of long bones between the joint and the primary growth plate in children. The epiphysis is initially completely comprised of cartilage with a high concentration of GAG, but ossifies during development $(4,5)$. By late adolescence, the epiphysis is typically completely ossified. During ossification GAG macromolecules degrade and the chondrocytes scattered in the epiphysis hypertrophy, reducing the amount of bound water. This transformation gives rise to higher signal on $\mathrm{T}_{2}$-weighted images (6).

There has been much recent interest in developing new imaging methods to identify the disease state of tissues with large quantities of exchanging protons and to relate them to quantitative relaxation parameters. Proton exchange between water and labile groups in other molecules provides one such potential mechanism that introduces sensitivity for specific chemical components within a mixture. This may be particularly useful for substances such as GAG, which contain a large concentration of chemically exchanging hydroxyl groups. While methods that monitor magnetization transfer such as chemical exchange saturation transfer (CEST) provide direct measurement of exchange (7), other approaches, notably $T_{2}$ and $T_{1 \rho}$ sequences, are also affected by exchange on appropriate time scales and register as large dispersions in signal contrast (8). Measurements of relaxation time constants in the rotating frame $\left(\mathrm{T}_{1 \rho}\right.$ and $\left.\mathrm{T}_{2 \rho}\right)$ using spin-locking techniques have been shown to be sensitive to molecular motions and chemical exchange on the time scale of the locking field $\left(\gamma B_{1}\right)(9,10)$. The advantage of the $T_{1 \rho}$ technique lies in the ability to make dispersion measurements in an imaging context in a regime where other approaches, such as CEST or Carr-Purcell-Meiboom-Gill (CPMG) dispersion, may be technically difficult $(11,12)$. For example, it is technically much easier to achieve high locking field strength $(>1 \mathrm{kHz})$ than to use comparable CPMG pulse spacing, making spin locking more appropriate for use in an imaging context. Spin locking techniques typically involve the application of a long, low power $\mathrm{B}_{1}$ prepulse before an imaging sequence to impart $\mathrm{T}_{1 \rho}$ contrast. The first experiments measuring relaxation in the rotating frame are attributed to Redfield, and Lee and Goldburg $(13,14)$. These types of measurements can, in principle, yield insights into the time scale of molecular motions, the sizes of different pools of protons, chemical and diffusive exchange processes, protein sizes and concentrations, and other attributes of interest $(10,15-17)$. $\mathrm{T}_{1 \rho}$ contrast has previously been investigated in tissues such as cartilage, brain, breast, and muscle (18-21). However, there is little consensus on the relative contribution of chemical exchange and other changes in tissue composition and their interactions with water on quantitative measures of $T_{1 \rho}$.

Articular cartilage has been studied in vivo with MRI using a variety of 2D and 3D methods, and articular cartilage degradation in adults has recently been assessed with $\mathrm{T}_{1 \rho}$-weighted imaging $(20,22-25)$. These studies report that early osteoarthritis (OA) changes are associated with the loss of PG and collagen, although early state molecular degradation is not typically seen on standard MRI using spin-echo and gradient recalled echo imaging sequences. $T_{2}$ and $T_{1 \rho}$ maps of articular cartilage have been made by several researchers 
while attempting to correlate proteoglycan degradation in OA to changes in $T_{1 \rho}$ values $(23,26)$. A competing technique, delayed Gadolinium-enhanced magnetic resonance imaging of cartilage (dGEMRIC), is quantitatively sensitive to PG loss, but requires the use of an intravenous contrast agent (27). It would, therefore, be preferable, especially for children, to use an endogenous source of contrast such as the chemical exchange effects of labile protons on GAG. A variety of imaging studies have been performed to measure cartilage structural changes during the maturation process $(4,28,29)$, but to date no applications of spin-lock techniques have been reported in children to quantify these effects in vivo. Thus, the purpose of this study is to determine the feasibility of $T_{1 \rho}$ mapping of pediatric epiphyseal and articular cartilage.

\section{MATERIALS AND METHODS}

A total of 13 patient-volunteers were recruited to have $T_{1 \rho}$ mapping performed at the conclusion of their clinically indicated MRI studies. Out of this pool of volunteers, 10 subjects of age $6 \pm 3$ years ( 5 male, 5 female) were included in our study as their epiphyseal and articular cartilage appeared normal based on conventional anatomic MRI sequences that were performed before $\mathrm{T}_{1 \rho}$ mapping. A fat-saturated, $3 \mathrm{D} \mathrm{T}_{2}$-weighted turbo spin-echo (TSE) sequence was used for this purpose. The criteria for the determination of "normal cartilage tissue" included the absence of any obvious clinical abnormality, such as the presence of lesions, edema, or ligament tear in close proximity.

All study patients underwent safety screening and their guardians provided written informed consent in compliance with the Institutional Review Board. Subjects were imaged using a 3.0T MR scanner with an eight-channel knee coil (Philips Achieva 3T, Philips Healthcare). The structures imaged included the lateral condyle of the knee in a sagittal orientation, and the patella and trochlea in an axial orientation. For each orientation, a single-slice exam of one epiphysis was performed for $\mathrm{T}_{1 \rho}$ quantification to optimize image quality and speed as some patients were imaged under sedation. For example, although the knee contains three epiphyses (distal femur, proximal tibia, and proximal fibula), $\mathrm{T}_{1 \rho}$ imaging and quantification was performed only for the distal femur.

First, the clinical 3D $\mathrm{T}_{2}$-weighted TSE sequence was used for pathology screening and planning purposes. The same experienced pediatric musculoskeletal radiologist then identified a position in either a sagittal or axial orientation to maximize the cross-sectional area of articular and epiphyseal cartilage. For the distal femur, regions of interest (ROIs) were placed in non-load-bearing (NLB) and load-bearing (LB) areas of epiphyseal cartilage and also in articular cartilage (Fig. 1a). The cartilaginous area directly below the ossification center where the femoral head is in contact with the tibia was considered LB. The proximal, posterior region of the femoral head was considered NLB as viewed in the planning images. For the patella and trochlea, ROIs were placed in epiphyseal and articular cartilage (Fig. 2a). Given the large age and size differences among the subjects, it was impossible to use uniform ROI sizes and positions. The minimum ROI size was 18 pixels for the articular cartilage of the youngest subject.

$\mathrm{T}_{1 \rho}$ contrast was generated using a SL prepulse cluster developed by Zeng et al (30) shown in Figure 3. First, a $500 \mu \mathrm{s} 90$-degree hard pulse was applied about the x-axis. This was followed by half of the spin-lock pulse with a SLA of $500 \mathrm{~Hz}$ along the y-axis. A $1 \mathrm{~ms} 180$ degree hard pulse was then inserted to compensate for $\mathrm{B}_{0}$ and $\mathrm{B}_{1}$ inhomogeneities, followed by the second half of the spin-lock pulse with phase reversed $(-\mathrm{y})$. From there, another 90 degree hard pulse returned the $\mathrm{T}_{1 \rho}$-prepped signal to the longitudinal axis, and the residual transverse magnetization was spoiled. A standard 2D TSE was then used for imaging. TSE parameters included: acquired matrix $=160 \times 256$, field of view $=100 \times 120 \mathrm{~mm}$, slice 
thickness $=4 \mathrm{~mm}$, echo-train length $=6, \mathrm{TE}=10 \mathrm{~ms}, \mathrm{TR}=4000 \mathrm{~ms}$, pixel bandwidth $=$ $\sim 155 \mathrm{~Hz}$. Spin-lock times of 20,40,60, and $80 \mathrm{~ms}$ were acquired using the same transmitter and receiver gains among acquisitions.

Each $\mathrm{T}_{1 \rho}$-weighted scan took approximately $1 \mathrm{~min}$ and $20 \mathrm{~s}$ to complete, for approximately a 5 -min scan time to compute a $T_{1 \rho}$ map. $T_{1 \rho}$ maps were generated by fitting each pixel of the images in the $\mathrm{T}_{1 \rho}$-weighted scans to a three-parameter mono-exponential decay function in MATLAB (The MathWorks, Inc., Natick, MA). A 120 ms maximum threshold was applied to the $\mathrm{T}_{1 \rho}$ map values and the patella, and soft tissue regions were removed for clarity.

A one-way analysis of variance (ANOVA) was performed to determine if a statistically significant difference exists among the group means. Student's t-tests were then performed to compare the mean $T_{1 \rho}$ values in each region. The Bonferroni post hoc multiple comparisons procedure was used to reduce the allowed $P$ value for each successive comparison to ensure an overall significance level of less than 0.05. All statistical calculations were performed in MATLAB.

\section{RESULTS}

$\mathrm{T}_{1 \rho}$ maps were successfully calculated for each of the 10 subjects. Figures $1 \mathrm{a}$ and $1 \mathrm{~b}$ show a representative sagittal planning scan with ROIs marked along with a corresponding map of $\mathrm{T}_{1 \rho}$ values overlaid on the planning image. Figures $2 \mathrm{a}$ and $2 \mathrm{~b}$ show a representative axial planning scan, also with overlaid ROIs and matching $T_{1 \rho}$ map overlay. Mean values in the lateral condyle of the femur for articular, LB, and NLB epiphyseal cartilage values are shown in Figure 4a. Additionally, LB and NLB epiphyseal cartilage values were averaged to give an overall epiphyseal cartilage value for each subject. For the patella and trochlea, mean values from articular and epiphyseal ROIs are shown in Figure 4b. Finally, average fitted $\mathrm{T}_{1 \rho}$ values for each anatomic region are summarized in Table 1.

One-way ANOVA was performed for ROIs in the lateral condyle across subjects and a significant difference was found in the underlying group means $(P<0.0006)$. Student's ttests, assuming an unequal variance, were then performed to compare means between pairs of the ROIs. Significant differences were found between articular versus LB epiphyseal, articular versus NLB epiphyseal, and articular versus the average epiphyseal cartilage value. All $P$ values were less than 0.0001 . No significant difference could be established $(P>0.02)$ in a comparison of LB, NLB, and average epiphyseal means given the more stringent $P$ value requirements for Bonferroni corrected multiple-comparison testing.

For the patellar and trochlear regions, a significant difference was found among the underlying articular and epiphyseal cartilage means $(P=0.0002)$. Student's t-tests revealed a significant difference between patellar epiphyseal and patellar articular cartilage $(P=$ 0.003); however, a comparison of trochlear articular and trochlear epiphyseal cartilage was not considered significant $(P=0.36)$.

When comparing $T_{1 \rho}$ values with age, no simple linear trends were noticeable across all ROIs and ages. No trends in $\mathrm{T}_{1 \rho}$ values versus sex were noted either. However, within each subject, average epiphyseal relaxation rates $\left(R_{1 \rho}=1 / T_{1 \rho}\right)$ always exceeded articular cartilage rates. Visual inspection of the average articular cartilage $R_{1 \rho}$ values plotted with average epiphyseal values versus age showed an intriguing parabolic trend shown in Figure 5. A simple second order polynomial was used to fit the young and old articular cartilage data, (Eq. [1]) it yielded a reasonable fit for the articular group $\left(\mathrm{R}^{2}=0.73\right)$ and an even better fit $\left(R^{2}=0.88\right)$ for the epiphyseal cartilage data. 


$$
R_{1 \rho}(x)=a x^{2}+b x+c
$$

Where $\mathrm{x}$ is age and the constants $\mathrm{a}, \mathrm{b}$, and $\mathrm{c}$ are allowed to freely vary to fit the data and have no known or implied physiologic meaning.

The fits suggest that maximum relaxation rates for epiphyseal and articular cartilage may be expected between the ages of 6 and 7 years.

\section{DISCUSSION}

The purpose of this study was to determine the feasibility of measuring $T_{1 \rho}$ values in young cartilage and to distinguish between cartilage regions in children. The $T_{1 \rho}$ measurement technique described here is a feasible method for differentiating epiphyseal and articular cartilage in a pediatric population. Epiphyseal cartilage composition has previously been compared with articular cartilage in $\mathrm{T}_{2}$-weighted images (28). This study establishes that these differences are also visible and are quantitatively different based on fitted $\mathrm{T}_{1 \rho}$ parameter maps.

Extensive laboratory and clinical research has been performed attempting to validate the utility of $\mathrm{T}_{1 \rho}$ in assessing articular cartilage and the study of osteoarthritis in adults $(20,22,26,31)$. To our knowledge, this is the first study that attempts in vivo $T_{1 \rho}$ imaging of epiphyseal cartilage. Epiphyseal cartilage is an important precursor to epiphyseal ossification; perturbations in its normal development may have profound effects on joint function and may lead to early osteoarthritis. Epiphyseal cartilage composition is uniquely different from articular cartilage in that it has a greater concentration of GAG, is more loosely structured, is infiltrated by vascular channels, and is present in far greater quantities than articular cartilage depending on skeletal maturation $(4,5)$. Cartilage cell hypertrophy and vacuolization occurs during normal epiphyseal skeletal maturation and reflects the dynamic and unique nature of epiphyseal cartilage compared with articular cartilage whose morphology is more static. The limited resolution of the scans and wide range of ages studied precluded systematic evaluation of epiphyseal or articular zonal variation. A larger study with a higher image resolution may be sensitive to these features, and therefore the $\mathrm{T}_{1 \rho}$ pulse sequence may need to be tailored accordingly to maximize sensitivity to the features of this cartilage type. Despite the small study size and heterogeneous age of patients, epiphyseal cartilage $T_{1 \rho}$ values were relatively similar across age ranges.

Artifacts related to $\mathrm{B}_{1}$ and $\mathrm{B}_{0}$ inhomogeneity are commonly seen in $\mathrm{T}_{1 \rho}$-weighted imaging techniques especially at low spin-lock amplitudes (32). The use of a self-compensating SL pulse in this study mitigated these effects so that banding artifacts were relegated to the edges of the field of view. However, self-compensating pulses do not perfectly counteract $B_{1}$ and $B_{0}$ inhomogeneity as the spins may not be perfectly refocused by the 180-degree pulse or rewound by the phase inversion. Thus $\mathrm{B}_{1}$ and $\mathrm{B}_{0}$ inhomogeneity could alter the effective locking field applied across the sample ROIs and thus affect the measured $\mathrm{T}_{1 \rho}$ values.

Alternative methods of quantifying articular cartilage quality by MRI have been studied, including $\mathrm{T}_{2}$ mapping and dGEMRIC $(27,33,34)$. These methods may be useful for evaluating articular cartilage, but may have limited utility in the evaluation of epiphyseal cartilage. Epiphyseal cartilage uniquely has prominent vascular channels, a property not seen in articular cartilage (4). These channels may falsely indicate a low level of GAGs using the dGEMRIC technique with the intravenous administration of Gadolinium, although these effects have yet to be quantitatively established. Quantitative $T_{2}$ and measurements 
will also be affected by the presence of water channels, where changes in water content may be difficult to distinguish from chemical exchange effects from changes in the concentration of GAG. For these reasons, optimizing $T_{1 \rho}$ measurement techniques to the study of chemical exchange effects in epiphyseal cartilage may be valuable as a method to monitor variations in tissue composition. At high fields the increased separation of resonance frequencies between water and other chemical species such as hydroxyls on GAG gives rise to greater contributions to $T_{1 \rho}$ decay from chemical exchange. These exchange processes are of interest not only for their effects on $\mathrm{T}_{1 \rho}$ but also on $\mathrm{T}_{2}$ and as modulators of saturation transfer contrast (e.g., CEST) (17,35). There have been few studies undertaken which attempt to quantify these processes or derive parameters that describe them in biological samples, and these findings represent an important first step toward quantification of these processes in vivo.

No significant differences in $\mathrm{T}_{1 \rho}$ values were observed in load- and non-load-bearing epiphyseal cartilage regions in the knee. This may be attributed to the wide age range and other developmental differences in the cartilage of the population studied. Previous reports of differences in $T_{2}$ between LB and NLB epiphyseal cartilage in piglets have been attributed to desiccation as water is compressed out of the LB tissue volume (36). $\mathrm{T}_{1 \rho}$ values may also be affected by free water content and/or by GAG composition changes due to variability between load and non- load-bearing sites. $T_{1 \rho}$ dispersion measurements, where the measured value of $T_{1 \rho}$ varies with locking field, may be required to distinguish between these effects. Appropriate model fitting of $\mathrm{T}_{1 \rho}$ dispersion curves may be used to distinguish the effects of variations in water content, GAG concentration, $\mathrm{pH}$, or disease state $(8,26)$. However, these quantitative methods are not widely used and have not yet been applied to epiphyseal cartilage in vivo.

Presenting normative epiphyseal cartilage $T_{1 \rho}$ values based on the stage of epiphyseal cartilage development will be important for comparison to perturbations in $\mathrm{T}_{1 \rho}$ values induced by pathology. Our findings differed from adult studies of $\mathrm{T}_{1 \rho}$ values in articular cartilage. The pediatric articular cartilage $\mathrm{T}_{1 \rho}$ values reported here are somewhat longer than those of healthy adult articular cartilage recently reported as $62 \pm 5 \mathrm{~ms}$ at $4 \mathrm{~T}$ (37) and $46 \pm 3$ $\mathrm{ms}$ at $3 \mathrm{~T}(24)$. However, $\mathrm{T}_{1 \rho}$ relaxation values in developing and mature articular cartilage may not be directly comparable. Numerous studies on developing cartilage illustrate the role of chondrocyte density, vascular canals, and epiphyseal vessels on young cartilage hydration $(5,38,39)$. This is in contrast to mature cartilage, which is typically avascular, and thus subject only to synovial hydration and thus may impart different mechanistic effects on $\mathrm{T}_{1 \rho}$ and $\mathrm{T}_{2}$. The large variance in articular cartilage $\mathrm{T}_{1 \rho}$ values is most likely related to the wide age range in developmental terms that also is likely reflected in changes in tissue structure and hydration status $(5,28)$. Trends in these tissue changes may be reflected in Figure 5, although these data and the polynomial fit should be interpreted with caution.

Three additional factors may contribute to uncertainty in the reported values. First, partial volume effects from noncartilage tissue or epiphyseal cartilage, if included in the ROI, may have affected the reported $\mathrm{T}_{1 \rho}$ values. Free water has a long $\mathrm{T}_{1 \rho}$ value and any contamination of the articular ROI from adjacent joint fluid would lengthen the reported value. Second, the locking field durations (TSL) that were used are mostly shorter than the average $\mathrm{T}_{1 \rho}$ times reported here. An ideal sampling scheme would allow for TSL values of at least two $\mathrm{T}_{1 \rho}$ time constants in length (40). However, this was deemed impractical due to specific absorption rate limitations. Finally, epiphyseal cartilage is also different from articular cartilage in terms of structure and of water content and its inclusion in an ROI would have an effect on the reported values. This is most likely reflected in the standard deviation of the articular cartilage values where there is less margin of error in the ROI boundaries between layers. 
In conclusion, $\mathrm{T}_{1 \rho}$ is a feasible method for quantifying differences in load- and non-loadbearing epiphyseal cartilage, and for differentiating epiphyseal and articular cartilage. $T_{1 \rho}$ holds promise as a noninvasive method of studying normal and abnormal developmental states of epiphyseal cartilage.

\section{Acknowledgments}

Contract grant sponsor: NIH; Contract grant number: R01-EB00214.

\section{References}

1. Shapiro EM, Borthakur A, Kaufman JH, Leigh JS, Reddy R. Water distribution patterns inside bovine articular cartilage as visualized by $1 \mathrm{H}$ magnetic resonance imaging. Osteoarthritis Cartilage. 2001; 9:533-538. [PubMed: 11520167]

2. Carney SL, Muir H. The structure and function of cartilage proteoglycans. Physiol Rev. 1988; 68:858-910. [PubMed: 3293094]

3. Huber M, Trattnig S, Lintner F. Anatomy, biochemistry, and physiology of articular cartilage. Invest Radiol. 2000; 35:573-580. [PubMed: 11041151]

4. Jaramillo D, Connolly SA, Mulkern RV, Shapiro F. Developing epiphysis: MR imaging characteristics and histologic correlation in the newborn lamb. Radiology. 1998; 207:637-645. [PubMed: 9609885]

5. Jaramillo D, Villegas-Medina OL, Doty DK, et al. Age-related vascular changes in the epiphysis, physis, and metaphysis: normal findings on gadolinium-enhanced MRI of piglets. AJR Am J Roentgenol. 2004; 182:353-360. [PubMed: 14736661]

6. Rivas R, Shapiro F. Structural stages in the development of the long bones and epiphyses: a study in the New Zealand white rabbit. J Bone Joint Surg Am. 2002; 84:85-100. [PubMed: 11792784]

7. Ling W, Regatte RR, Navon G, Jerschow A. Assessment of glycosaminoglycan concentration in vivo by chemical exchange-dependent saturation transfer (gagCEST). Proc Natl Acad Sci U S A. 2008; 105:2266-2270. [PubMed: 18268341]

8. Duvvuri U, Goldberg AD, Kranz JK, et al. Water magnetic relaxation dispersion in biological systems: the contribution of proton exchange and implications for the noninvasive detection of cartilage degradation. Proc Natl Acad Sci U S A. 2001; 98:12479-12484. [PubMed: 11606754]

9. Hills BP. The proton-exchange cross-relaxation model of water relaxation in biopolymer systems. Mol Phys. 1992; 76:489-508.

10. Hills BP. The proton-exchange cross-relaxation model of water relaxation in biopolymer systems. 2. The Sol and Gel states of gelatin. Mol Phys. 1992; 76:509-523.

11. van Zijl PCM, Yadav NN. Chemical exchange saturation transfer (CEST): what is in a name and what isn't? Magn Reson Med. 2011; 65:927-948. [PubMed: 21337419]

12. Meiboom S, Gill D. Modified spin-echo method for measuring nuclear relaxation times. Rev Sci Instrum. 1958; 29:688-691.

13. Redfield AG. Nuclear magnetic resonance saturation and rotary saturation in solids. Physical Rev. $1955 ; 98: 1787$.

14. Lee M, Goldburg WI. Nuclear-magnetic-resonance line narrowing by a rotating Rf field. Phys Rev. 1965; 140:1261-1271.

15. Henkelman RM, Huang X, Xiang QS, Stanisz GJ, Swanson SD, Bronskill MJ. Quantitative interpretation of magnetization transfer. Magn Reson Med. 1993; 29:759-766. [PubMed: 8350718]

16. Koenig SH, Brown RD III, Ugolini R. A unified view of relaxation in protein solutions and tissue, including hydration and magnetization transfer. Magn Reson Med. 1993; 29:77-83. [PubMed: 8380481]

17. Gore JC, Brown MS, Zhong J, Mueller KF, Good W. NMR relaxation of water in hydrogel polymers: a model for tissue. Magn Reson Med. 1989; 9:325-332. [PubMed: 2709998]

18. Santyr GE, Henkelman RM, Bronskill MJ. Spin locking for magnetic resonance imaging with application to human breast. Magn Reson Med. 1989; 12:25-37. [PubMed: 2607958] 
19. Aronen HJ, Ramadan UA, Peltonen TK, et al. 3D spin-lock imaging of human gliomas. Magn Reson Imaging. 1999; 17:1001-1010. [PubMed: 10463651]

20. Regatte RR, Akella SV, Wheaton AJ, et al. 3D-T1rho-relaxation mapping of articular cartilage: in vivo assessment of early degenerative changes in symptomatic osteoarthritic subjects. Acad Radiol. 2004; 11:741-749. [PubMed: 15217591]

21. Virta A, Komu M, Lundbom N, Kormano M. T1 rho MR imaging characteristics of human anterior tibial and gastrocnemius muscles. Acad Radiol. 1998; 5:104-110. [PubMed: 9484543]

22. Akella SV, Regatte RR, Gougoutas AJ, et al. Proteoglycan-induced changes in T1rho-relaxation of articular cartilage at 4T. Magn Reson Med. 2001; 46:419-423. [PubMed: 11550230]

23. Menezes NM, Gray ML, Hartke JR, Burstein D. T2 and T1rho MRI in articular cartilage systems. Magn Reson Med. 2004; 51:503-509. [PubMed: 15004791]

24. Li X, Benjamin Ma C, Link TM, et al. In vivo T(1rho) and T(2) mapping of articular cartilage in osteoarthritis of the knee using 3 T MRI. Osteoarthritis Cartilage. 2007; 15:789-797. [PubMed: 17307365]

25. Souza RB, Stehling C, Wyman BT, et al. The effects of acute loading on T1rho and T2 relaxation times of tibiofemoral articular cartilage. Osteoarthritis Cartilage. 2010; 18:1557-1563. [PubMed: 20950693]

26. Regatte RR, Akella SV, Borthakur A, Reddy R. Proton spin-lock ratio imaging for quantitation of glycosaminoglycans in articular cartilage. J Magn Reson Imaging. 2003; 17:114-121. [PubMed: 12500280]

27. Gray ML, Burstein D, Kim YJ, Maroudas A. 2007 Elizabeth Winston Lanier Award Winner. Magnetic resonance imaging of cartilage glycosaminoglycan: basic principles, imaging technique, and clinical applications. J Orthop Res. 2008; 26:281-291. [PubMed: 17876836]

28. Laor T, Jaramillo D. MR imaging insights into skeletal maturation: what is normal? Radiology. 2009; 250:28-38. [PubMed: 19092089]

29. Xia Y, Moody JB, Alhadlaq H, Hu J. Imaging the physical and morphological properties of a multi-zone young articular cartilage at microscopic resolution. J Magn Reson Imaging. 2003; 17:365-374. [PubMed: 12594728]

30. Zeng, H. A composite spin-lock pulse for delta B0 and B1 insensitive T1rho measurements. Proceedings of the 14th Annual Meeting of ISMRM; Seattle. 2006. p. abstract 2356

31. Burstein D, Bashir A, Gray ML. MRI techniques in early stages of cartilage disease. Invest Radiol. 2000; 35:622-638. [PubMed: 11041156]

32. Witschey WR Jr, Borthakur A, Elliott MA, et al. Artifacts in T1 rho-weighted imaging: compensation for B(1) and B(0) field imperfections. J Magn Reson. 2007; 186:75-85. [PubMed: 17291799]

33. Trattnig S, Marlovits S, Gebetsroither S, et al. Three-dimensional delayed gadolinium-enhanced MRI of cartilage (dGEMRIC) for in vivo evaluation of reparative cartilage after matrix-associated autologous chondrocyte transplantation at 3. 0T: preliminary results. J Magn Reson Imaging. 2007; 26:974-982. [PubMed: 17896385]

34. McKenzie CA, Williams A, Prasad PV, Burstein D. Three-dimensional delayed gadoliniumenhanced MRI of cartilage (dGEMRIC) at 1.5T and 3. 0T. J Magn Reson Imaging. 2006; 24:928933. [PubMed: 16941612]

35. Zhou JY, Payen JF, Wilson DA, Traystman RJ, van Zijl PCM. Using the amide proton signals of intracellular proteins and peptides to detect pH effects in MRI. Nat Med. 2003; 9:1085-1090. [PubMed: 12872167]

36. Jaramillo D, Connolly SA, Vajapeyam S, et al. Normal and ischemic epiphysis of the femur: diffusion MR imaging study in piglets. Radiology. 2003; 227:825-832. [PubMed: 12773684]

37. Regatte RR, Akella SV, Lonner JH, Kneeland JB, Reddy R. T1rho relaxation mapping in human osteoarthritis (OA) cartilage: comparison of T1rho with T2. J Magn Reson Imaging. 2006; 23:547-553. [PubMed: 16523468]

38. Menezes NM, Olear EA, Li X, et al. Gadolinium-enhanced MR images of the growing piglet skeleton: ionic versus nonionic contrast agent. Radiology. 2006; 239:406-414. [PubMed: 16641351] 
39. Menezes NM, Connolly SA, Shapiro F, et al. Early ischemia in growing piglet skeleton: MR diffusion and perfusion imaging. Radiology. 2007; 242:129-136. [PubMed: 17185664]

40. Skinner MG, Kolind SH, MacKay AL. The effect of varying echo spacing within a multiecho acquisition: better characterization of long T2 components. Magn Reson Imaging. 2007; 25:840847. [PubMed: 17418518] 


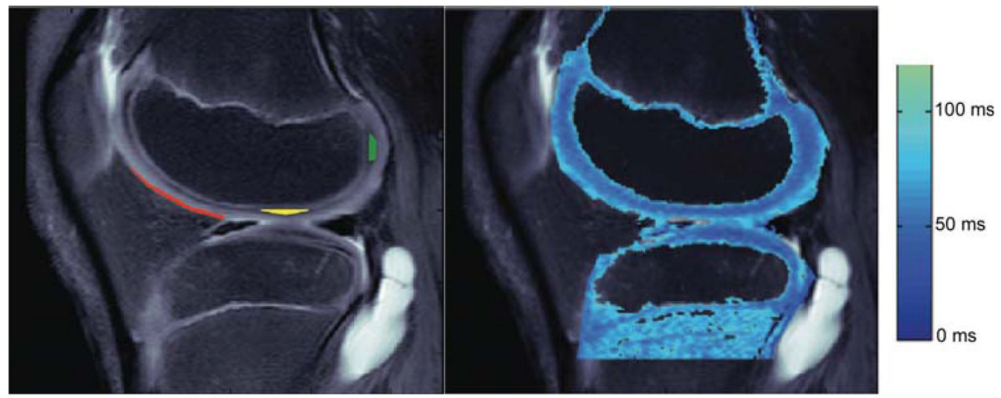

Figure 1.

a: A T2-weighted turbo spin echo (TSE) sagittal scan of the knee used to plan ROI for the $\mathrm{T}_{1 \rho}$ map shown in Figure 1b. The red ROI is articular cartilage, the yellow ROI is LB epiphyseal cartilage, and the green ROI is NLB epiphyseal cartilage. $\mathbf{b}$ : $\mathrm{T}_{1 \rho}$ map of the same subject as shown in Figure 1a. A maximum threshold of $120 \mathrm{~ms}$ was applied to the $\mathrm{T}_{1 \rho} \mathrm{map}$, and the soft tissue and patellar regions were removed for clarity. The color scale is given in ms. Note approximately a $25 \mathrm{~ms}$ difference in articular and epiphyseal cartilage $\mathrm{T}_{1 \rho}$ values. 

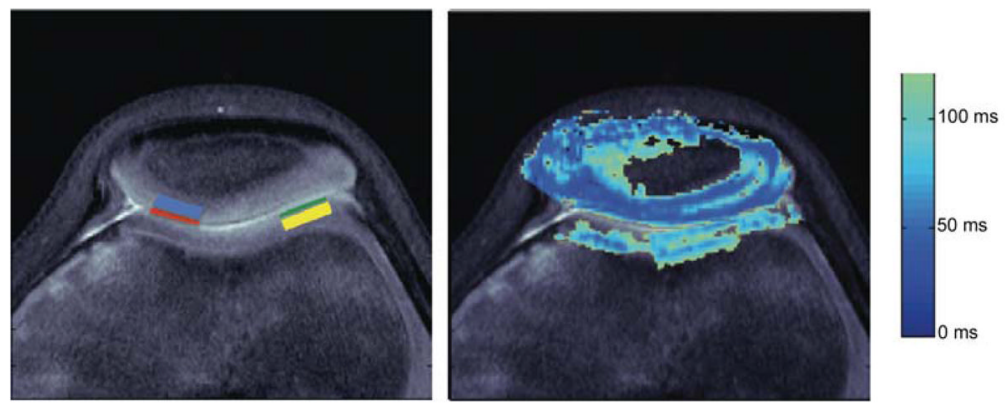

Figure 2.

a: T2-weighted turbo spin echo (TSE) axial scan of the trochlea and patella used to plan ROI for the $\mathrm{T}_{1 \rho}$ map in Figure $2 \mathrm{~b}$. The red ROI is the patellar articular cartilage, the blue ROI is the patellar epiphyseal cartilage, the green ROI is the trochlear articular cartilage, and the yellow ROI is the trochlear epiphyseal cartilage. Uniform $\mathrm{T}_{1 \rho}$ maps were more difficult to achieve in this orientation as evidenced by the low signal intensity in the lateral trochea. This region was avoided when placing ROI. b: $\mathrm{T}_{1 \rho}$ map of the same subject as shown in Figure $2 \mathrm{~b}$. A maximum threshold of $120 \mathrm{~ms}$ was applied to the $\mathrm{T}_{1 \rho}$ map, and the soft tissue regions were removed for clarity. The color scale is also given in ms. Note approximately a $25 \mathrm{~ms}$ difference in articular and epiphyseal cartilage $\mathrm{T}_{1 \rho}$ values. 

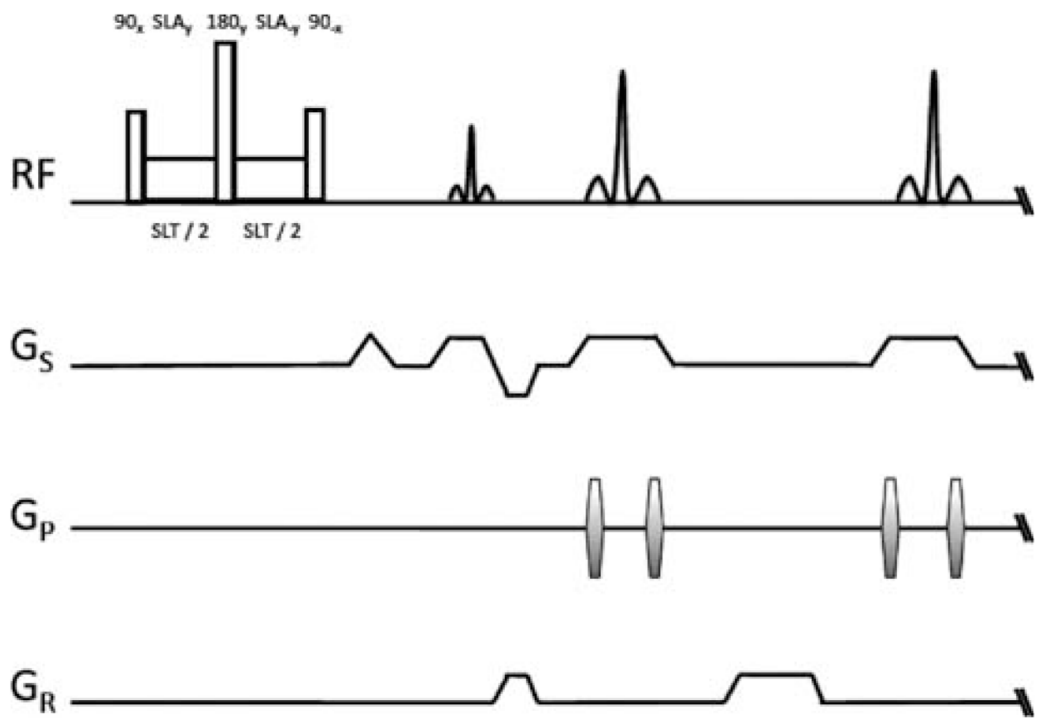

Figure 3.

Pulse sequence used to record $\mathrm{T}_{1 \rho}$-weighted fast spin-echo images. 

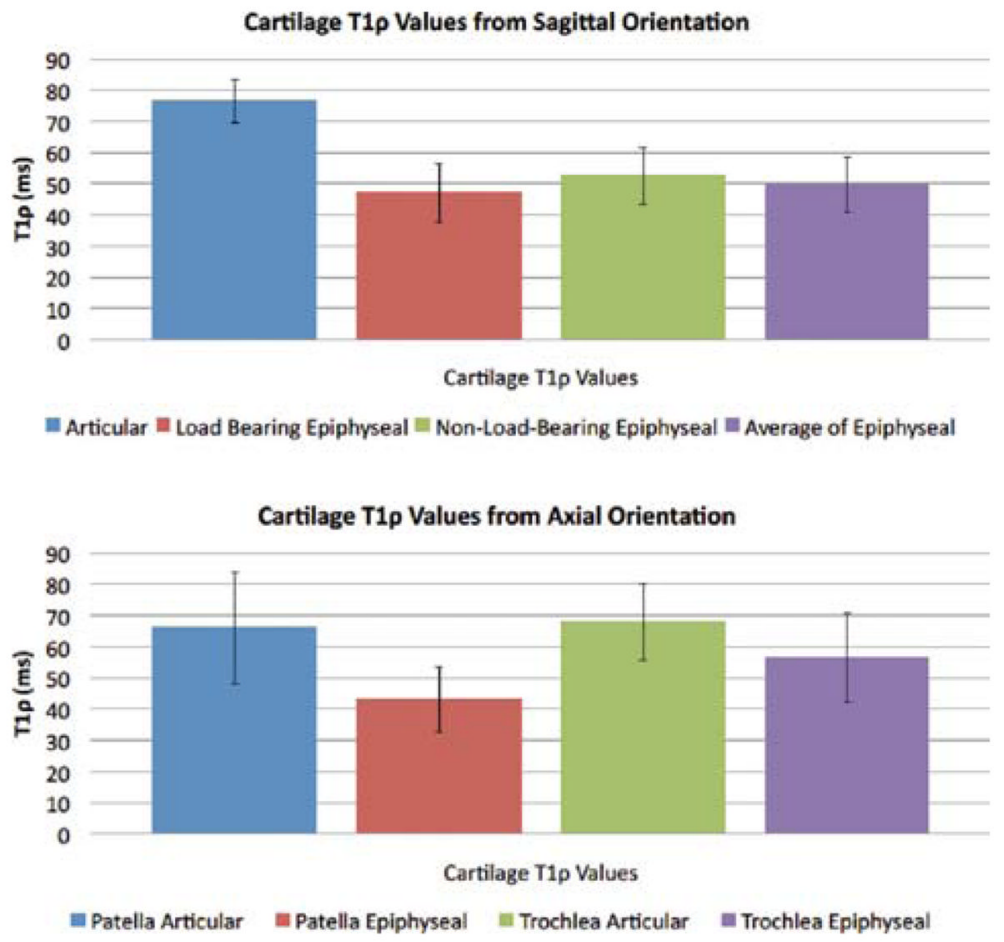

Figure 4.

a: Distal femur cartilage $\mathrm{T}_{1 \rho}$ values at $3 \mathrm{~T}(\mathrm{~ms} \pm \mathrm{SD})$. Articular $=76.6 \pm 6.9$, Load-bearing epiphyseal $=47.1 \pm 9.5$, non-load-bearing epiphyseal $=52.5 \pm 8.9$, average epiphyseal $=$ $49.8 \pm 9.0$. b: patella and trochlear cartilage $\mathrm{T}_{1 \rho}$ values at $3 \mathrm{~T}(\mathrm{~ms} \pm \mathrm{SD})$. Patella articular $=$ $66.1 \pm 17.8$, patella epiphyseal $=43.0 \pm 10.6$, trochlea articular $=67.9 \pm 14.3$, trochlea epiphyseal $=56.7 \pm 12.3$. [Color figure can be viewed in the online issue, which is available at wileyonlinelibrary.com.] 


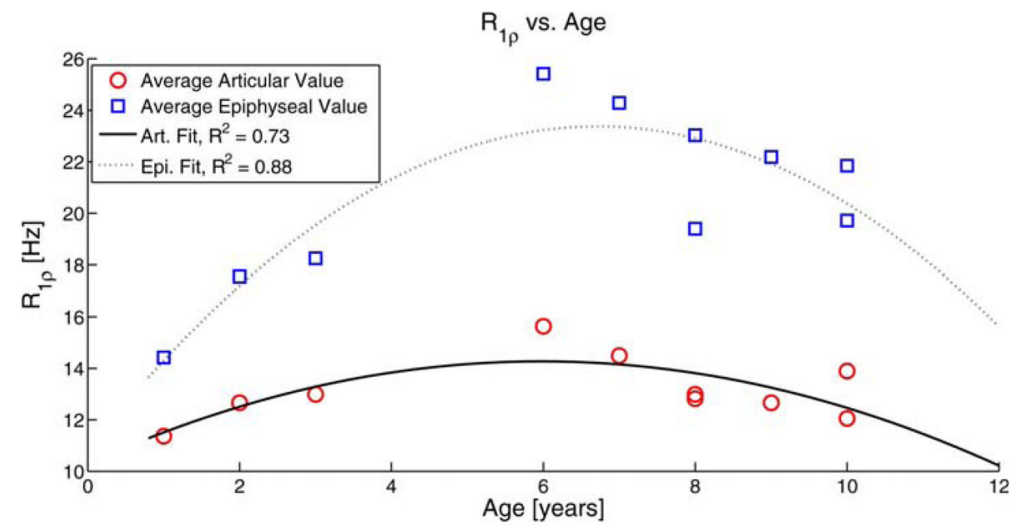

Figure 5.

Plot of cartilage $R_{1 \rho}$ values $\left(1 / T_{1 \rho}\right)$ versus age at $3 T$. Note the epiphyseal cartilage relaxation rates always exceed the articular cartilage relaxation rates. [Color figure can be viewed in the online issue, which is available at wileyonlinelibrary.com.] 


\section{Table 1}

Average $\mathrm{T}_{1 \rho}$ Values for the Lateral Condyle, Patella, and Trochlea in Children Using a $500 \mathrm{~Hz}$ SL Pulse, Measured at 3 Tesla* $^{*}$

\begin{tabular}{lll}
\hline \multicolumn{2}{c}{ Anatomical region } & Average \pm SD $\mathbf{T}_{\mathbf{1} \boldsymbol{\rho}}$ value $(\mathbf{m s})$ \\
\hline Lateral condyle & Articular & $76.6 \pm 6.9$ \\
& NLB epiphyseal & $52.5 \pm 8.9$ \\
& LB epiphyseal & $47.1 \pm 9.5$ \\
& Average epiphyseal & $49.8 \pm 9.0$ \\
Patella & Articular & $66.2 \pm 17.8$ \\
& Epiphyseal & $43.3 \pm 10.6$ \\
Trochlea & Articular & $67.9 \pm 14.3$ \\
& Epiphyseal & $56.7 \pm 12.3$ \\
\hline
\end{tabular}

* $\mathrm{EB}=$ load bearing epiphyseal cartilage, $\mathrm{NLB}=$ non-load bearing epiphyseal cartilage, $\mathrm{SD}=$ standard deviation. 